\title{
El polimorfismo 5-HTTLPR y el intento suicida en adolescentes deprimidos
}

\author{
Emmanuel I. Sarmiento Hernández, ${ }^{1}$ Rosa Elena Ulloa Flores, ${ }^{2}$ Mirna Esthela Brenes Prats, ${ }^{1}$ \\ Beatriz Camarena Medellín, ${ }^{3}$ Alejandro Aguilar García, ${ }^{3}$ Sandra Hernández Muñoz, ${ }^{3}$
}

Artículo original

\section{SUMMARY}

Suicide is a common cause of death in adolescents, being mainly associated with depression. In addition, the "SS" genotype and the "S" allele of 5-HTTLPR polymorphism of SLC6A4 gene of serotonin transporter have been associated with suicidal behavior. The aims of the present study were to compare the frequency of the polymorphism of SLC6A4 gene in depressed adolescents with and without history of suicidal attempt and to determine if the "SS" genotype was associated with specific clinical features.

\section{Method}

The study examined 53 adolescents who were evaluated with the Diagnostic Interview Schedule for Affective Disorders and Schizophrenia for school-aged children-present and lifetime version (K-SADS-PL). A DNA sample was obtained and 5HTTLPR polymorphisms of SLC6A4 gene were analyzed.

\section{Results}

There were no differences in the frequency of genotype and allele frequencies between groups. However, patients with the "SS" genotype reported a higher frequency of hopelessness and a greater number of suicide attempts.

\section{Conclusions}

The frequency of "SS" genotype did not differ between patients with and without suicidal behavior, but patients with this genotype exhibited differences in clinical features of depression which need further study.

Key words: Adolescents, suicide, depression, genetics.

\section{RESUMEN}

La Organización Mundial de la Salud reporta que el suicidio es la tercera causa de muerte más frecuente para jóvenes de 15 a 24 años de edad y la sexta causa de muerte para niños de cinco a 14 años de edad. Los trastornos del estado de ánimo, particularmente la depresión, son los responsables de la mayor parte de los suicidios consumados. Este mayor riesgo de suicidio se ha encontrado en adultos y adolescentes. Existe cada vez mayor evidencia respecto de la hipótesis de que la conducta suicida tiene una fuerte contribución genética. Varios estudios han reportado una asociación positiva entre el genotipo "SS" y el alelo "S" del polimorfismo 5-HTTLPR del gen del transportador de serotonina y la conducta suicida.

\section{Objetivo}

El objetivo del presente trabajo fue establecer la asociación de las variantes polimórficas del gen del transportador de serotonina en pacientes adolescentes deprimidos con y sin antecedente de intento suicida y determinar si la presencia del genotipo "SS" estaba asociada a características específicas de la depresión.

\section{Método}

La muestra estuvo conformada por 53 adolescentes con diagnóstico de depresión. El diagnóstico se realizó con la entrevista diagnóstica semi-estructurada K-SADS-PL. Para la extracción del ADN genómico se obtuvo una muestra de sangre de cada uno de los pacientes.

\section{Resultados}

El análisis genético de las frecuencias de genotipos y alelos no mostró diferencias estadísticamente significativas entre los grupos. Sin embargo, aquellos pacientes con el genotipo "SS" tenían mayor frecuencia de desesperanza. En los pacientes con este genotipo también se encontró mayor número de intentos suicidas.

\section{Conclusiones}

No se observaron diferencias en la frecuencia de alelos entre pacientes con y $\sin$ intento suicida; sin embargo, el genotipo "SS" se asoció a algunas características de la depresión.

Palabras clave: Adolescentes, suicidio, depresión, genética.

Servicio de Consulta Externa. Hospital Psiquiátrico Infantil "Juan N. Navarro".

Departamento de Psicofarmacología del Desarrollo. Hospital Psiquiátrico Infantil "Juan N. Navarro".

Subdirección de Investigaciones Clínicas. Instituto Nacional de Psiquiatría Ramón de la Fuente Muñiz (INPRFM).

Correspondencia: Dra. Rosa Elena Ulloa Flores. Psicofarmacología del Desarrollo, Hospital Psiquiátrico Infantil "Juan N. Navarro". San Buenaventura 86, Belisario Domínguez, Tlalpan, 14080, México, DF. E-mail: eulloa@hotmail.com

Recibido primera versión: 1ro. de marzo de 2012. Segunda versión: 19 de marzo de 2013. Tercera versión: 23 de septiembre de 2013. Aceptado: 14 de noviembre de 2013. 


\section{INTRODUCCIÓN}

Aunque el suicidio explica casi el 2\% de las muertes en todo el mundo, el intento de suicidio es más frecuente durante el curso de la vida con una prevalencia cercana al 3.5\%, estimándose que de ese porcentaje hasta el 10\% terminará en suicidio consumado en un intervalo de 10 años. ${ }^{1}$ Según las estimaciones de la Organización Mundial de la Salud, para el año 2020 aproximadamente un millón y medio de personas fallecerán por suicidio y de 15 a 30 millones cometerán un intento. ${ }^{2}$ La misma organización establece que actualmente el suicidio se encuentra dentro de las cinco principales causas de mortalidad entre los 15 y 19 años de edad y es la sexta causa de muerte para aquellos de entre cinco a 14 años de edad. ${ }^{3}$

\section{SUICIDIO EN ADOLESCENTES}

Varios reportes sugieren que el suicidio en la gente joven está aumentando en muchas áreas geográficas., ${ }^{4,5}$ Por ejemplo, en Estados Unidos se reporta que aproximadamente ocho a $10 \%$ de los adolescentes han tenido en algún momento de su vida algún intento suicida. ${ }^{6}$

En lo que respecta a México, en el 2007 se reportaron 4388 muertes por suicidio en toda la República, con una tasa de mortalidad por suicidio de 4.12 por cada cien mil habitantes, lo cual representa un incremento del $275 \%$ respecto a la tasa de suicido reportada en 1970. Lo que llama aún más la atención de este dato es que este incremento es especialmente marcado en la población joven. ${ }^{7}$

En el año 2010, un estudio transversal realizado en 12424 adolescentes de escuelas públicas de educación media superior pertenecientes a los 32 Estados de México, reportó que 47\% de los estudiantes encuestados habían tenido ideación suicida en algún momento de su vida. Además, 9\% de los estudiantes reportaron el antecedente de, por lo menos, un intento de suicidio. ${ }^{8}$

\section{PSICOPATOLOGÍA Y SUICIDIO}

Frecuentemente el suicidio se asocia con muchos trastornos psiquiátricos, como por ejemplo los trastornos afectivos, el abuso de sustancias y algunos trastornos de la personalidad. En personas con tales condiciones, el riesgo de suicidio es 10 a 20 veces superior que en la población general. ${ }^{2}$

Varios investigadores han propuesto que los trastornos del estado de ánimo, particularmente la depresión, son los responsables de la mayor parte de los suicidios consumados tanto en adultos como en adolescentes., ${ }^{9,10}$ En este último grupo más de la mitad eventualmente realizará un intento de suicidio, y aproximadamente el 10\% terminará en un suicidio consumado dentro de los 15 años posteriores a su diagnóstico. ${ }^{11,12}$

\section{GENÉTICA Y SUICIDIO}

Durante los últimos años se han realizado numerosos esfuerzos dirigidos a establecer las bases genéticas del comportamiento suicida. De acuerdo con Bondy et al., se estima que el $43 \%$ de la variabilidad en la conducta suicida pudiera ser explicada por la genética, mientras que el restante $57 \%$ lo es por factores ambientales. ${ }^{1}$

Varios estudios moleculares han buscado asociaciones para los genes de las enzimas, los transportadores y las proteínas del receptor de serotonina [5-hidroxitriptamina (5-HT)] requeridos para la neurotransmisión sináptica. ${ }^{13} \mathrm{Al}$ respecto, existe evidencia de que el gen del transportador de serotonina (SLC6A4) está asociado con la conducta suicida. ${ }^{14}$ Se trata de una proteína clave en la regulación de la neurotransmisión serotoninérgica al ser responsable de la recaptación sináptica de la serotonina y el lugar de acción de numerosos antidepresivos. Este gen se localiza en el brazo largo del cromosoma 17 (17q11.112); en dicho gen se tiene identificado un polimorfismo en la región promotora denominado 5-HTTLPR, el cual consiste en la inserción/deleción de 44 pares de bases, dando lugar a dos alelos, una forma corta o "S" (deleción) y la variante larga o "L" (inserción). Diversos estudios han encontrado una asociación positiva entre el genotipo "SS" y el alelo " $S$ " del polimorfismo 5-HTTLPR y el suicidio consumado, los intentos de suicidio y una historia familiar de conducta suicida. ${ }^{15,16}$

Por lo que respecta a la población adolescente, algunos estudios han analizado el polimorfismo 5-HTTLPR y su relación con la conducta suicida obteniendo distintos resultados. En un estudio realizado por Zalsman et al. en 48 pacientes de 16 a 24 años de edad evaluados mediante los instrumentos K-SADS-PL y SCID, no se encontró una asociación que fuera significativa entre el polimorfismo 5-HTTLPR y la conducta suicida, sin embargo sí hubo una asociación significativa entre la presencia de conductas violentas en adolescentes suicidas y el genotipo LL del transportador de serotonina. ${ }^{17}$ En otro estudio realizado recientemente por el mismo autor en 60 adolescentes, también evaluados con el K-SADSPL (32 con intento suicida y 28 sin intento suicida), no se encontraron diferencias significativas al analizar este mismo polimorfismo. ${ }^{18}$

En nuestro país la cifra de suicidio en niños y adolescentes se ha incrementado considerablemente en la última década. A pesar de ello, existen relativamente pocas investigaciones dirigidas a estudiar dicho fenómeno. El objetivo del presente trabajo fue establecer la asociación de las variantes polimórficas del gen del transportador de serotonina en pacientes adolescentes deprimidos con y sin antecedente de intento suicida y determinar si la presencia del genotipo "SS" estaba asociada a características específicas de la depresión. 


\section{MATERIAL Y METODOS}

El reclutamiento de los pacientes se realizó de forma consecutiva durante el periodo comprendido entre agosto y diciembre del 2010 en los servicios de consulta externa, hospitalización y urgencias del Hospital Psiquiátrico Infantil "Dr. Juan N. Navarro". Se incluyeron a todos los pacientes de 12 a 17 años de edad con diagnóstico de depresión con y sin antecedente de intento suicida, sin importar la comorbilidad ni el tratamiento recibido. La aceptación para participar en el estudio se realizó por medio de la firma del consentimiento informado por el paciente y su padre o tutor.

A todos los pacientes se les aplicó la entrevista diagnóstica semi-estructurada Schedule for affective disorders and schizophrenia for school-aged children-present and lifetime version (K-SADS-PL), en su versión validada al español. ${ }^{19}$

El K-SADS-PL fue adaptado del K-SADS-P, el cual fue desarrollado por William Chambers y Joaquin Puig-Antich. Dicha entrevista fue diseñada para evaluar episodios actuales y pasados de psicopatología en niños y adolescentes de acuerdo a los criterios del DSM-III-R y el DSM-IV.

Después de completar la entrevista se tomó una muestra de sangre para la obtención y análisis de los genotipos y alelos mediante reacción en cadena de la polimerasa, utilizando las condiciones descritas por Camarena et al. ${ }^{20}$

Para la descripción de características clínicas y demográficas se utilizaron frecuencias y porcentajes para las variables categóricas, y medias y desviación estándar (DE)

Cuadro 1. Comorbilidad de los pacientes deprimidos

\begin{tabular}{|c|c|c|c|}
\hline Diagnóstico comórbido & $\begin{array}{l}\text { Paciente } \\
\text { con I.S. }\end{array}$ & $\begin{array}{l}\text { Paciente } \\
\text { sin I.S. }\end{array}$ & $\begin{array}{l}\text { Porcentaje } \\
\text { total }\end{array}$ \\
\hline Distimia & 0 & 1 & 1.8 \\
\hline $\begin{array}{l}\text { Trastorno de ansiedad } \\
\text { generalizada }\end{array}$ & 3 & 9 & 22.6 \\
\hline Trastorno de pánico & 0 & 1 & 1.8 \\
\hline Agorafobia & 0 & 1 & 1.8 \\
\hline Ansiedad de separación & 0 & 6 & 11.3 \\
\hline Fobia simple & 0 & 3 & 5.6 \\
\hline Fobia social & 1 & 8 & 16.9 \\
\hline $\begin{array}{l}\text { Trastorno por déficit de atención } \\
\text { e hiperactividad }\end{array}$ & 6 & 11 & 32.1 \\
\hline $\begin{array}{l}\text { Trastorno oposicionista } \\
\text { desafiante }\end{array}$ & 4 & 12 & 30.2 \\
\hline Trastorno disocial & 0 & 1 & 1.8 \\
\hline Alcohol & 2 & 0 & 3.7 \\
\hline Cannabis & 0 & 1 & 1.8 \\
\hline Inhalantes & 0 & 1 & 1.8 \\
\hline Bulimia & 1 & 2 & 5.6 \\
\hline Enuresis & 0 & 1 & 1.8 \\
\hline Encopresis & 0 & 1 & 1.8 \\
\hline Trastorno de estrés postraumático & 3 & 1 & 7.5 \\
\hline
\end{tabular}

I.S.= Intento suicida. para las variables continuas. Como pruebas de hipótesis en la comparación de los pacientes se utilizó una Chi Cuadrada $\left(\chi^{2}\right)$ para contrastes categóricos y se calcularon riesgos no ajustados. También se utilizó la prueba $t$ de Student para comparación de promedios. El nivel de significancia estadística se fijó con una $\mathrm{p} \leq 0.05$.

\section{RESULTADOS}

Se incluyeron 53 pacientes de los cuales el $54.7 \%$ pertenecía al sexo femenino $(n=29)$. La edad promedio fue de $14.25 \pm 1.7$ años (intervalo 12-17 años).

El 22.6\% ( $n=12)$ presentó depresión como único diagnóstico, el 26.4\% ( $\mathrm{n}=14)$ presentó un trastorno comórbido, el 34\% $(n=18)$ presentó dos, el 11.3\% $(n=6)$ presentó tres y, finalmente, el $5.7 \%(n=3)$ presentó cuatro trastornos comórbidos. En el cuadro 1 se muestra la distribución de los principales diagnósticos comórbidos de los pacientes con y sin antecedente de intento suicida y los porcentajes totales de la muestra.

De la totalidad de la muestra, el 26.4\% (n=14) presentó, al menos, un intento suicida claro de acuerdo al K-SADS-PL (calificación de 3, tanto en la severidad como en la letalidad). En la figura 1 se muestran los principales métodos empleados en el último intento suicida reportado por los pacientes.

Encontramos algunas diferencias significativas en la frecuencia de algunos síntomas del suplemento de depresión del K-SADS-PL entre pacientes suicidas y no suicidas. En particular, en el primer grupo hubo mayor frecuencia de disminución del apetito (57.1\% vs. $28.2 \%, \mathrm{x}^{2}=7.3, \mathrm{gl}=2$, $\mathrm{p}=0.025)$, pérdida de peso $\left(21.4 \%\right.$ vs. $12.8 \%, \mathrm{x}^{2}=10.24$, $\mathrm{gl}=2$,

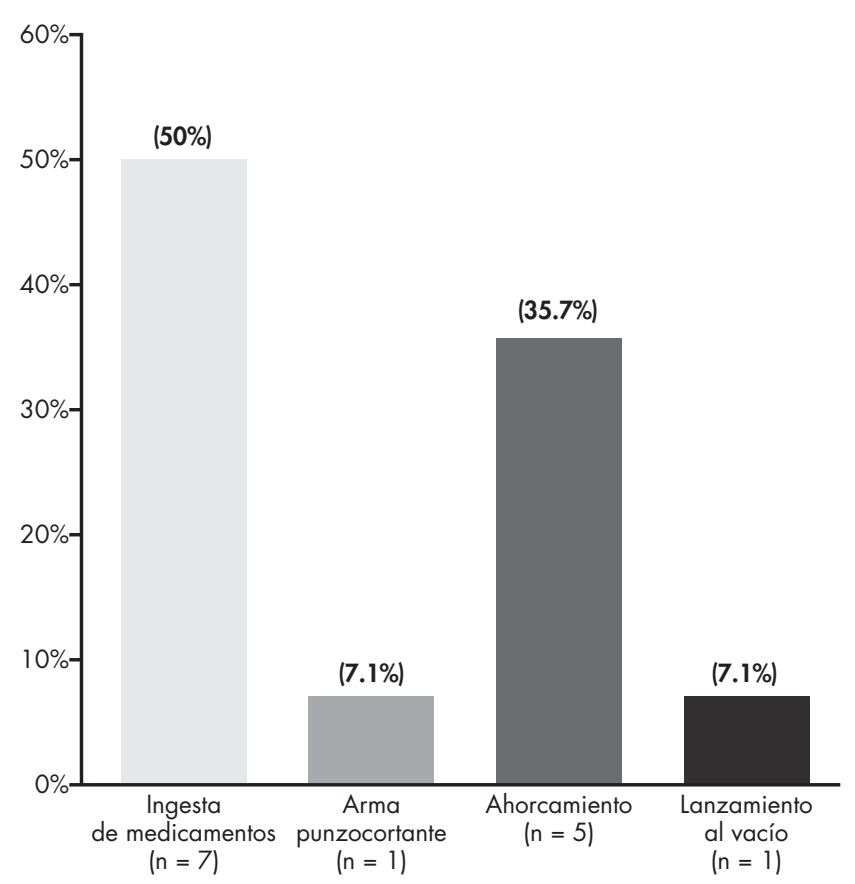

Figura 1. Método utilizado en el intento suicida. 
Cuadro 2. Frecuencia de genotipos y alelos del polimorfismo 5 HTTLPR del gen SLC6A4

\begin{tabular}{llllllll}
\hline & \multicolumn{3}{c}{ Genotipos } & & \multicolumn{3}{c}{ Alelos } \\
\cline { 2 - 6 } Depresión & SS & SL & $\mathrm{LL}$ & & $\mathrm{S}$ & $\mathrm{L}$ \\
\hline
\end{tabular}

Con intento suicida $5(0.36) \quad 7(0.50) 2(0.14) 17(0.61) 11(0.39)$

Sin intento suicida 13(0.33)18(0.46) $8(0.21) 44(0.56) 34(0.44)$

Genotipos: $X^{2}=0.26, g l=2, p=0.87$

Alelos: $X^{2}=0.15, g l=1, p=0.69$

$\mathrm{p}=0.006)$ y sensibilidad al rechazo $\left(71.4 \%\right.$ vs. $41 \%, \mathrm{x}^{2}=6.82$, $\mathrm{gl}=2, \mathrm{p}=0.03$ ).

El análisis de las frecuencias de los genotipos y los alelos no mostró diferencias significativas entre los grupos (cuadro 2). Sin embargo, aquellos pacientes con el genotipo "SS" tenían una mayor frecuencia de desesperanza $(66.7 \%$ vs. $31.4 \%, x^{2}=6,1$ gl, $\left.\mathrm{p}=0.014\right)$, (RM=4.36, IC 95\% 1.29-14.66). En los pacientes con este genotipo también se encontró mayor número de intentos suicidas $(1.33 \pm 1.23$ vs. $0.66 \pm 0.68$, $\mathrm{t}=2.57,51 \mathrm{gl}, \mathrm{p}=.013)$.

\section{DISCUSIÓN}

La literatura médica internacional ha reportado que del 40 al $70 \%$ de los adolescentes que sufren depresión presentan dos o más diagnósticos comórbidos, siendo los más frecuentes la distimia, los trastornos de ansiedad, el trastorno por déficit de atención e hiperactividad, los trastornos de conducta disruptiva y el abuso de sustancias. ${ }^{21}$ Los resultados de nuestro estudio no son la excepción ya que el $77.4 \%$ de la muestra tuvo uno o más trastornos comórbidos siendo los tres principales el trastorno por déficit de atención e hiperactividad, el trastorno negativista desafiante y el trastorno por ansiedad generalizada.

Dentro del grupo suicida, mención especial merece la presencia del trastorno de estrés postraumático, ya que en nuestro estudio tres de los cuatro casos diagnosticados reportaron haber cometido un intento suicida. Investigaciones en este tema reportan que las personas que tienen el diagnóstico de trastorno de estrés postraumático tienen 2.7 veces más probabilidades de tener algún intento suicida en comparación con el resto de la población. ${ }^{22}$

En este estudio las variables clínicas de pérdida del apetito y perdida del peso, así como la sensibilidad al rechazo, fueron los síntomas que más frecuentemente se asociaron con la presencia de intentos suicidas. Si bien la disminución importante en el apetito y la subsecuente pérdida de peso son características clínicas reportadas constantemente por los pacientes con diagnóstico de depresión, hasta nuestro conocimiento no existen estudios que reporten la asociación de estas dos características clínicas con la presencia de un intento suicida; sin embargo, tomando en cuenta que ambas forman parte del diagnóstico de depresión atípica, su presencia en adolescentes suicidas podría formar parte de un subtipo distinto de depresión en este grupo de edad. Respecto a la presencia de una mayor sensibilidad al rechazo, también poco se sabe sobre su relación con las conductas suicidas. Es por esto que deberán llevarse a cabo más estudios al respecto en nuestro medio con la finalidad de evaluar cuáles son las características clínicas de la depresión en adolescentes que intentan suicidarse, en comparación con los que no lo intentan.

En relación con los métodos suicidas utilizados, tampoco hubo diferencias con estudios que analizan esta variable. Como regla general, los métodos más violentos y letales como el ahorcamiento se utilizan en los suicidios consumados, mientras que los métodos no violentos como la ingesta de fármacos se suelen preferir en los intentos de suicidio. ${ }^{23,24}$ En nuestra muestra la ingesta de medicamentos fue el método más utilizado, reportándose en el 50\% de los casos.

En nuestro estudio no encontramos una asociación entre las variantes del polimorfismo 5-HTTLPR y el antecedente de intento suicida, lo cual repite los hallazgos de no asociación realizados en niños y adolescentes. ${ }^{25,26}$ Sin embargo, encontramos una asociación de un mayor número de intentos suicidas en sujetos con genotipo SS. Lo anterior se podría explicar, en parte, debido a que la presencia de este genotipo ha sido asociada con mayores niveles de impulsividad y agresividad. ${ }^{27}$

En cuanto a la comparación del presente estudio con otros realizados en población clínica de adolescentes, nuestros hallazgos son semejantes a los resultados de no asociación reportados recientemente, en donde se encontraron frecuencias de genotipos similares. ${ }^{18} \mathrm{El}$ fenotipo de intento suicida es altamente heterogéneo, por lo que analizar rasgos asociados con dicha conducta permite tener características más homogéneas que ayuden a encontrar los genes de susceptibilidad para el desarrollo de conductas tan complejas como el suicidio, como lo demuestra el metaanálisis de Clayden, el cual reporta que no existe correlación entre los polimorfimos genéticos y la conducta suicida en general; sin embargo encuentra una asociación significativa entre los subgrupos de personas con intento suicida y el gen transportador de serotonina 5-HTTLPR. ${ }^{16}$

Por otra parte, el análisis de las características clínicas mostró un hallazgo interesante en los pacientes portadores del genotipo "SS", los cuales presentaron una mayor gravedad de desesperanza. Este hecho apoya los datos que muestran que los pacientes con este síntoma presentan un mayor riesgo de tener intento suicida. ${ }^{28,29}$ Es interesante resaltar que este resultado es similar al obtenido por Kangelaris et al., en donde se reportó que la desesperanza tuvo una asociación significativa con el alelo " $S$ " del 5-HTTLPR, independientemente del diagnóstico de depresión, concluyendo que el ser portador de dicho alelo pudiera incrementar la susceptibilidad a desarrollar este síntoma. ${ }^{30}$

Si bien los resultados obtenidos no demuestran una asociación significativa entre el gen estudiado y la conducta suicida, la asociación encontrada entre el genotipo SS con 
el número de intentos suicidas y la desesperanza, así como la presencia de variables como pérdida del apetito, pérdida de peso y sensibilidad al rechazo en el grupo con intento suicida, nos podría indicar la existencia de un grupo de adolescentes con un subtipo de depresión grave y que son portadores de la variante $S$ del transportador de serotonina.

Para finalizar, debemos mencionar que la principal limitación de nuestro estudio fue el tamaño y la selección de la muestra, por lo que los hallazgos reportados en el presente estudio deben de ser tomados como preliminares hasta que sean replicados en una muestra de mayor tamaño. Del mismo modo, es posible que debido a que los estudios de asociación son susceptibles a presentar problemas de estratificación poblacional, nos encontremos ante un falso positivo, aunque resulta interesante que las frecuencias alélicas observadas en nuestro estudio son similares a las reportadas en un estudio epidemiológico de adolescentes mexicanos. ${ }^{31}$ En futuros estudios será necesario que se lleve a cabo el análisis de ésta u otras variantes genéticas en muestras de mayor tamaño en un intento de clarificar el posible papel que juega este gen en la conducta suicida.

\section{CONCLUSIÓN}

A pesar de que en la actualidad el suicidio en adolescentes es un importante problema de salud pública, los estudios genéticos al respecto continúan siendo escasos. Este estudio incrementa la información sobre la conducta suicida en adolescentes mexicanos, correlacionando las características clínicas con un genotipo específico.

\section{REFERENCIAS}

1. Bondy B, Buettner A, Zill P. Genetics of suicide. Molecular Psychiatry 2006;11:336-351.

2. Bertolote JM, Fleishman A. A global perspective in the epidemiology of suicide. Suicidology 2002;7:6-8.

3. Ventura-Juncá DR, Carvajal C, Undurraga $S$, Vicuña $P$ et al. Prevalence of suicidal ideations and suicidal attempts among adolescents living in Metropolitan Santiago. Rev Med Chile 2010;138:309-315.

4. Brent DA, Oquendo M, Birmaher B, Greenhill L et al. Peripubertal Suicide Attempts in offspring of suicide attempters with siblings concordant for suicidal behavior. Am J Psychiatry 2003;160(8):1486-1493.

5. Baldessarini RJ, Hennen J. Genetics of suicide: An overview. Harv Rev Psychiatry 2004;12(1):1-13.

6. Miller M. Firearm prevalence and the risk of suicide: a review. Harvard Health Policy Review 2000;2:13.

7. Borges G, Orozco R, Benjet C, Medina-Mora ME. Suicide and suicidal behaviors in Mexico: Retrospective and current status. Salud Publica Mex 2010;52:292-304.

8. Pérez-Amezcua B, Rivera-Rivera L, Atienzo EE, De Castro F et al. Prevalencia y factores asociados a ideación e intento suicida en estudiantes de México. Salud Pública México 2010;52(4):324-333.

9. Bostwick JM, Pankratz VS. Affective disorders and suicide risk: a reexamination. Am J Psychiatry 2000;157:1925-1932.

10. Bradvik L, Berglund M. Suicidal ideation in severe depresión. Eur Arch Psychiatry Clin Neurosci 2000;250(3):139-143.

11. Keith CR. Adolescent suicide: perspectives on a clinical quandary. JAMA 2001;286:3126-3127.
12. Pfeffer CR. Diagnosis of childhood and adolescent suicidal behavior: unmet needs for suicide prevention. Soc Biol Psychiatry 2001;49:1055-1061.

13. Pitchot $W$ et al. 5-Hydroxytryptamine $1 A$ receptors, major depression, and suicidal behavior. Biological Psychiatry 2005;58:854-858.

14. Gonga X, Fountoulakis KN, Harro J, Pompili M et al. The possible contributory role of the s allele of 5-HTTLPR in the emergence of suicidality. J Psychopharmacol 2011;25(7):857-866.

15. Li D, He L. Meta-analysis supports association between serotonin transporter (5-HTT) and suicidal behavior. Molecular Psychiatry 2007;12:47-54.

16. Clayden RC, Zaruk A, Meyre D, Thabane $L$ et al. The association of attempted suicide with genetic variants in the SLC6A4 and TPH genes depends on the definition of suicidal behavior: a systematic review and meta-analysis. Transl Psychiatry 2012;2:1-8.

17. Zalsman G, Frisch A, Bromberg M, Gelernter J et al. Family-based association study of serotonin transporter promoter in suicidal adolescents: no association with suicidality but possible role in violence traits. Am J Med Genet 2001;105:239-245.

18. Zalsman G, Anderson GM, Peskin M, Frisch A et al. Relationships between serotonin transporter promoter polymorphism, platelet serotonin transporter binding and clinical phenotype in suicidal and non-suicidal adolescent inpatients. J Neural Transmission 2005;112(2):309-315.

19. Ulloa R, Ortíz S, Higuera F, Nogales I et al. Estudio de fiabilidad interevaluador de la versión en español de la entrevista Schedule for affective disorders and schizophrenia for school-aged children-present and lifetime version (K-SADS-PL). Actas Esp Psiquiatr 2006;34(1):36-40.

20. Camarena $B$ et al. Association study of the serotonin transporter gene polymorphism in obsessive-compulsive disorder. International J Neuropsychopharmacology 2001;4:269-272.

21. Rao U, Chen LA. Characteristics, correlates and out comes of child and adolescent depressive disorders. Dialogues clin neurosci 2009; 11(1):45-62.

22. Gradus JL, Qin P, Lincoln AK, Miller M et al. Posttraumatic stress disorder and completed suicide. Am J Epidemiol 2010;171:721-727.

23. Tsirigotis K, Gruszczynski W, Tsirigotis-Woloszczak M. Gender differentiation in methods of suicide attempts. Med Sci Monit 2011;17(8):65-70.

24. Kposowa AJ, McElvain JP. Gender, place, and method of suicide. Soc Psychiatry Psychiatr Epidemiol 2006;41(6):435-443.

25. Zalsman G. Timing is critical: Gene, environment and timing interactions in genetics of suicide in children and adolescents. European Psychiatry 2010;25:284-286.

26. Dante Cicchetti D, Rogosch F, Sturge-Apple M, Toth SL. Interaction of child maltreatment and 5-HTT polymorphisms: Suicidal ideation among children from low-SES backgrounds. J Pediatric Psychology 2010;35(5):536-546.

27. Jiménez-Treviño L, Blasco-Fontecilla $H$, Braquehais $M$, Ceverino-Dominguez A et al. Endophenotypes and suicide behaviour. Review. Actas Esp Psiquiatr 2011;39(1):61-69.

28. Chabrol H, Choquet M. Relationship between depressive symptoms, hopelessness and suicidal ideation among 1547 high school students. Encephale 2009;35(5):443-447.

29. Kwok SY, Shek DT. Hopelessness, parent-adolescent communication and suicidal ideation among Chinese adolescent in Hong-Kong. Suicide Life Threat Behav 2010;40(3):224-233

30. Kangelaris KN, Vittinghoff E, Otte C, Na B, Auerbach AD, Whooley MA. Association between a serotonin transporter gene variant and hopelessness among men in the heart and soul study. J Gen Intern Med 2010;25(10):1030-1037.

31. Martínez-Levy GA, Benjet C, Perez-Molina A, Gómez-Sánchez A et al. Association of the catechol-O-methyltransferase gene and attention deficit hyperactivity disorder: results from an epidemiological study of adolescents of Mexico City. Psychiat Genet 2013;23(2):90-91.

Artículo sin conflicto de intereses 